\title{
Enhanced cooling of neutron stars via Cooper-pairing neutrino emission
}

\author{
M. E. Gusakov ${ }^{1}$, A. D. Kaminker ${ }^{1}$, D. G. Yakovlev ${ }^{1}$, and O. Y. Gnedin ${ }^{2}$ \\ ${ }^{1}$ Ioffe Physical Technical Institute, Politekhnicheskaya 26, 194021 St. Petersburg, Russia \\ e-mail: [gusakov;kam;yak]@astro.ioffe.ru \\ 2 Space Telescope Science Institute, 3700 San Martin Drive, Baltimore, MD 21218, USA \\ e-mail: ognedin@stsci.edu
}

Received 1 April 2004 / Accepted 2 May 2004

\begin{abstract}
We simulate cooling of superfluid neutron stars with nucleon cores where the direct Urca process is forbidden. We adopt density-dependent critical temperatures $T_{\mathrm{cp}}(\rho)$ and $T_{\mathrm{cn}}(\rho)$ of singlet-state proton and triplet-state neutron pairing in a stellar core and consider strong proton pairing (with maximum $\left.T_{\mathrm{cp}}^{\max } \gtrsim 5 \times 10^{9} \mathrm{~K}\right)$ and moderate neutron pairing $\left(T_{\mathrm{cn}}^{\max } \sim\right.$ $6 \times 10^{8} \mathrm{~K}$ ). When the internal stellar temperature $T$ falls below $T_{\mathrm{cn}}^{\max }$, the neutrino luminosity $L_{\mathrm{CP}}$ due to Cooper pairing of neutrons behaves $\propto T^{8}$, just as that produced by the modified Urca process (in a non-superfluid star) but is higher by about two orders of magnitude. In this case the Cooper-pairing neutrino emission acts like an enhanced cooling agent. By tuning the density dependence $T_{\mathrm{cn}}(\rho)$ we can explain observations of cooling isolated neutron stars in the scenario in which the direct Urca process or a similar process in kaon/pion condensed or quark matter are absent.
\end{abstract}

Key words. stars: neutron - dense matter

\section{Introduction}

Thanks to the Chandra and XMM-Newton missions, there is great progress in observations of thermal radiation emergent from the surfaces of isolated (cooling) middle-aged neutron stars (e.g., Pavlov \& Zavlin 2003). A comparison of these data with theoretical models of cooling neutron stars gives a method to constrain the (still poorly known) fundamental properties of supranuclear matter in neutron-star cores, such as the composition and equation of state of the matter and its superfluid properties.

So far, the observations can be explained by a number of vastly different theoretical models (e.g., Page 1998a,b; Tsuruta et al. 2002; Khodel et al. 2004; Blaschke et al. 2004; Yakovlev $\&$ Pethick 2004, and references therein). Particularly, one can employ the simplest models of neutron stars with the cores composed of nucleons (or nucleons/hyperons), or containing pion condensates, kaon condensates or quarks. The simplest model of a non-superfluid nucleon core which cools via the modified Urca process of neutrino emission (without any powerful direct Urca process) cannot explain the observations: some neutron stars (e.g., PSR B1055-52) turn out to be much warmer, while others (e.g., the Vela pulsar) are much colder than those expected from this model. Warmer stars can be explained (Kaminker et al. 2001) assuming strong proton superfluidity in the core: such superfluidity suppresses the modified Urca process and slows down the cooling. However, colder stars require some cooling mechanism that is faster than the modified Urca process.

Explanations of observations of colder stars presented in the literature usually invoke either a powerful direct Urca process in nucleon (or nucleon/hyperon) matter or similar processes in kaon-condensed, pion-condensed, or quark matter in the inner cores of massive neutron stars.

In this paper we present a new scenario of neutron star cooling. We adopt the simplest model equation of state of supranuclear matter in neutron star cores (Douchin \& Haensel 2001) involving only nucleons, electrons and muons. This equation of state forbids the direct Urca process in all stable neutron stars. We will show that the enhanced cooling required to explain colder isolated neutron stars can be produced by neutrino emission due to moderately strong triplet-state pairing of neutrons. This new interpretation is possible only for a specific density dependence of the critical temperature of neutron pairing.

In the next section we outline the observational basis; the cooling scenario is given afterwards.

\section{Observations}

Table 1 summarizes observations of isolated (cooling) middleaged $\left(10^{3} \lessgtr t \lesssim 10^{6} \mathrm{yr}\right)$ neutron stars, whose thermal surface radiation has been detected (or constrained). We present the estimated stellar ages $t$ and effective surface temperatures $T_{\mathrm{s}}^{\infty}$ (as detected by a distant observer). 
Table 1. Observational limits on surface temperatures of isolated neutron stars.

\begin{tabular}{l|cccl}
\hline \hline Source & $t$ [kyr] & $T_{\mathrm{s}}^{\infty}[\mathrm{MK}]$ & Confid. & References \\
\hline PSR J0205+6449 & 0.82 & $<1.1^{b}$ & - & Slane et al. (2002) \\
Crab & 1 & $<2.0^{b}$ & $99.7 \%$ & Weisskopf et al. (2004) \\
RX J0822-4300 & $2-5$ & $1.6-1.9^{a}$ & $90 \%$ & Zavlin et al. (1999) \\
1E 1207.4-5209 & $3-20$ & $1.4-1.9^{a}$ & $90 \%$ & Zavlin et al. (2004) \\
Vela & $11-25$ & $0.65-0.71^{a}$ & $68 \%$ & Pavlov et al. (2001) \\
PSR B1706-44 & $\sim 17$ & $0.82_{-0.34}^{+0.01}$ & $68 \%$ & McGowan et al. (2004) \\
PSR J0538+2817 & $30 \pm 4$ & $\sim 0.87^{a}$ & - & Zavlin \& Pavlov (2003) \\
Geminga & $\sim 340$ & $\sim 0.5^{b}$ & $90 \%$ & Zavlin \& Pavlov (2003) \\
RX J1856.4-3754 & $\sim 500$ & $<0.65$ & - & see text \\
PSR B1055-52 & $\sim 540$ & $\sim 0.75^{b}$ & - & Pavlov \& Zavlin (2003) \\
RX J0720.4-3125 & $\sim 1300$ & $\sim 0.51^{a}$ & - & Motch et al. (2003) \\
\hline
\end{tabular}

${ }^{a}$ Inferred using a hydrogen atmosphere model.

${ }^{b}$ Inferred using the black-body spectrum.

Two young objects, RX J0822-4300 and 1E 1207.4-5209, are radio-quiet neutron stars in supernova remnants; RX J1856.4-3754 and RX J0720.4-3125 are also radioquiet neutron stars. Other objects - the Crab and Vela pulsars, PSR B1706-44, PSR J0538+2817, Geminga, and PSR B1055-52 - are observed as radio pulsars.

RX J0205+6449 and the Crab pulsar are associated with historical supernovae and their ages are certain. For RX J0822-4300, we take the age of the host supernova remnant, Puppis A. As can be deduced, e.g., from a discussion in Arendt et al. (1991), its age is between 2 to $5 \mathrm{kyr}$; the central value is $t=3.7 \mathrm{kyr}$ (Winkler et al. 1988). For 1E 1207.45209 , we also adopt the age of the host supernova remnant (G296.5+10). According to Roger et al. (1988), it is $t \sim$ 3-20 kyr. For the Vela pulsar, we take the age interval from the standard characteristic spindown age of the pulsar to the characteristic age corrected for the pulsar glitching behaviour (Lyne et al. 1996). The age of PSR J0538+2817, $t=(30 \pm 4) \mathrm{kyr}$, was estimated by Kramer et al. (2003) from the measurements of the pulsar proper motion relative to the center of the host supernova remnant, S147. The age of RX J1856.4-3754 has been revised recently by Walter \& Lattimer (2002) from the kinematics of proper motion. Following these authors we take the central value $t=500 \mathrm{kyr}$ and choose such an errorbar of $t$ that the revised value is clearly distinguished from the value $t=900$ kyr reported previously by Walter (2001) on the basis of less accurate parallax measurement. The characteristic age of RX J0720.4-3125 was estimated by Zane et al. (2002), Kaplan et al. (2002) and Cropper et al. (2004) from X-ray measurements of the neutron-star spindown rate. We adopt the central value $t=1300 \mathrm{kyr}$ with an uncertainty of a factor of 2 . The ages of three other pulsars, PSR B1706-44, Geminga, and PSR B1055-52, are the characteristic pulsar ages assuming an uncertainty of a factor of 2 .

For the two youngest sources, RX J0205+6449 and the Crab pulsar, no thermal emission has been detected, but upper limits on the surface temperature $T_{\mathrm{s}}^{\infty}$ have been established (Slane et al. 2002; Weisskopf et al. 2004). The surface temperatures of the next five sources, RX J0822-4300, 1E 1207.4-5209, Vela, PSR B1706-44, and PSR J0538+2817, have been obtained using hydrogen atmosphere models (see references in Table 1). Such models are more consistent with other information on these sources (e.g., Pavlov et al. 2002) than the blackbody model. On the contrary, for the Geminga and PSR B1055-52 we present the values of $T_{\mathrm{s}}^{\infty}$ inferred using the blackbody spectrum because this spectrum is more consistent for these sources.

Let us notice that from Table 1 we have excluded PSR B0656+14 which was considered earlier (e.g., Yakovlev et al. 2002). A combined analysis of new X-ray and optical observations of the source (with the improved distance from new parallax measurements of Brisken et al. 2003) leads either to unrealistically small values of the neutron star radius (in the blackbody model) or to an unreasonably small distance to the star (in the hydrogen atmosphere model); see, e.g., Zavlin \& Pavlov (2002). This makes current interpretations of the data unreliable.

The surface temperature of RX J1856.4-3754 is still rather uncertain. A wide scatter of $T_{\mathrm{s}}^{\infty}$, obtained by different authors, is present because X-ray and optical observations are not described by one blackbody model. This can be explained, for instance, by the presence of hot spots on the neutron star surface. Thus, we adopt the upper limit $T_{\mathrm{s}}^{\infty}<0.65 \mathrm{MK}$, which agrees with the value of $T_{\mathrm{s}}^{\infty}$ obtained either with the "Si-ash" atmosphere model of Pons et al. (2002) or with the model of condensed surface layers of Burwitz et al. (2003). It also agrees with the model of nonuniform surface temperature distribution suggested by Pavlov \& Zavlin (2003). In the latter case, the mean surface temperature $T_{\mathrm{s}}^{\infty} \approx 0.5 \mathrm{MK}$ is below our upper limit of $T_{\mathrm{s}}^{\infty}$.

Finally, $T_{\mathrm{s}}^{\infty}$ for RX J0720.4-3125 is taken from Motch et al. (2003) who have interpreted the observed spectrum with a model of a hydrogen atmosphere of finite depth.

For PSR J0538+2817, PSR B1055-52, and RX J0720.4-3125, the authors cited in Table 1 have not 

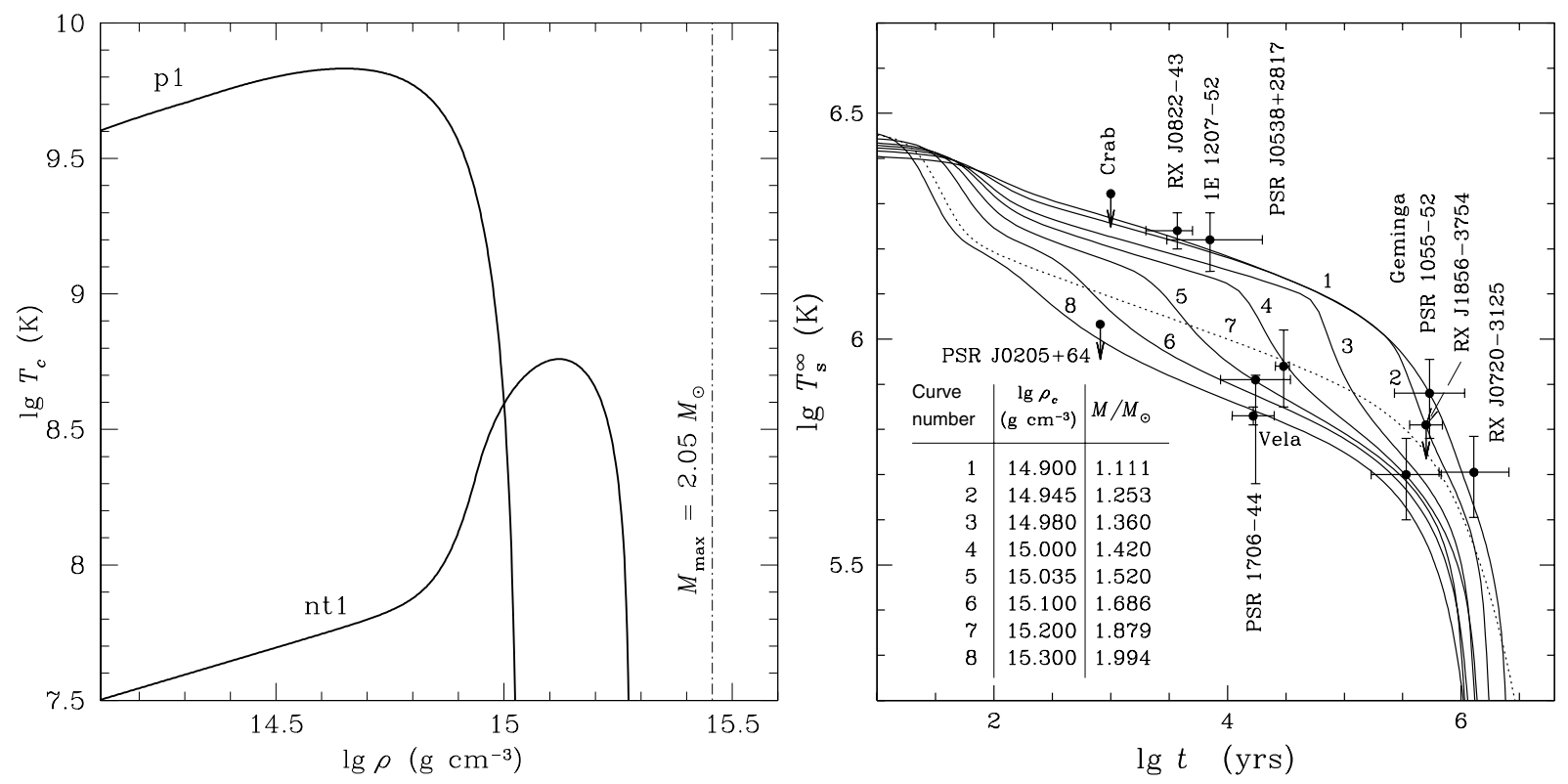

Fig. 1. Left: density dependence of the critical temperature of model p1 for proton superfluidity and model nt 1 for neutron superfluidity in a neutron-star core; the vertical dot-and-dash line indicates the central density of a maximum-mass neutron star. Right: observations (Table 1) compared with theoretical cooling curves of eight neutron stars (1-8) with different masses. All solid curves refer to neutron stars with model superfluidities from the left panel. The dotted curve 7 is for a non-superfluid star. The insert table gives masses and central densities of stars $1-8$.

reported any errors of $T_{\mathrm{s}}^{\infty}$. We adopt $20 \%$ uncertainties which seem to be appropriate for these sources.

\section{Physics input and calculations}

We will simulate the cooling of neutron stars using our general relativistic cooling code described by Gnedin et al. (2001). We adopt the moderately stiff equation of state of neutron star interiors proposed by Douchin \& Haensel (2001). According to this equation of state, neutron star cores (regions of density $\rho>1.3 \times 10^{14} \mathrm{~g} \mathrm{~cm}^{-3}$ ) consist of neutrons with the admixture of protons, electrons and muons. All constituents exist everywhere in the core, except for muons which appear at $\rho>2.03 \times 10^{14} \mathrm{~g} \mathrm{~cm}^{-3}$. The most massive stable star has (gravitational) mass $M=M_{\max }=2.05 M_{\odot}$, central density $\rho_{\mathrm{c}}=$ $2.9 \times 10^{15} \mathrm{~g} \mathrm{~cm}^{-3}$, and (circumferential) radius $R=9.99 \mathrm{~km}$. The central densities and masses of eight neutron star models (with $M$ from $1.111 M_{\odot}$ to $1.994 M_{\odot}$ ) are presented in the right panel of Fig. 1.

All physics input is standard. The effects of muons are included as described by Bejger et al. (2003). We assume no envelope of light elements on the stellar surfaces (Sect. 5). The code calculates the cooling curves, which give the dependence of the effective surface stellar temperature $T_{\mathrm{s}}^{\infty}$ on stellar age $t$. Let us recall that neutron stars are born hot in supernova explosions (with internal temperatures $T \sim 10^{11} \mathrm{~K}$ ) but gradually cool down via neutrino emission from the entire stellar body and via heat diffusion to the surface and thermal surface emission of photons. Qualitatively, one can distinguish three cooling stages. In the first ("non-isothermal") stage $(t \lesssim 100 \mathrm{yr}$ ) the main cooling mechanism is neutrino emission but the stellar interior stays highly non-isothermal. In the second ("neutrino") stage $\left(10^{2} \lesssim t \lesssim 10^{5} \mathrm{yr}\right)$ the cooling goes mainly via neutrino emission from isothermal interiors. In the third ("photon") stage ( $t \gtrsim 10^{5} \mathrm{yr}$ ) the star cools predominantly through surface photon emission.

The new element of our present studies is the equation of state of Douchin \& Haensel (2001). We have chosen it because it forbids the powerful direct Urca process of neutrino emission (Lattimer et al. 1991) in all stable neutron stars $\left(M \leq M_{\max }\right)$. In this case, a non-superfluid neutron star of any mass $M_{\odot} \lesssim M \leq M_{\max }$ will have almost the same (universal) cooling curve $T_{\mathrm{s}}^{\infty}(t)$ (the dotted curve in the right panel of Fig. 1). In the neutrino cooling stage, this curve is determined by the neutrino emission due to the modified Urca process. The curve is almost independent of the equation of state of neutron star cores (Page \& Applegate 1992) as long as the direct Urca process is forbidden. As has been indicated by many authors (see, e.g., Yakovlev \& Pethick 2004, and references therein) and seen from Fig. 1, this universal cooling model is certainly unable to explain the data. For instance, it gives $T_{\mathrm{s}}^{\infty}$ much lower than that of PSR B1055-52, but much higher than that of the Vela pulsar. We will show that all the data can be explained assuming superfluidity of neutron-star cores.

It is well known that neutrons and protons in stellar cores can be in the superfluid state. Proton superfluidity is caused by singlet-state proton pairing, while neutron superfluidity is produced by triplet-state neutron pairing. These superfluidities can be specified by density dependent critical temperatures for protons and neutrons, $T_{\mathrm{cp}}(\rho)$ and $T_{\mathrm{cn}}(\rho)$. The results of calculations of these temperatures from microscopic theories show a large scatter of critical temperatures depending on the nucleon-nucleon interaction model and the many-body theory employed. In particular, recently Schwenk \& Friman (2004) 
and Zuo et al. (2004) obtained weak neutron and proton pairing in neutron star cores but many other calculations give much stronger superfluidity (e.g., Lombardo \& Schulze 2001; see also references in Yakovlev et al. 1999). In this situation it is reasonable to consider $T_{\mathrm{cp}}(\rho)$ and $T_{\mathrm{cn}}(\rho)$ as unknown functions of $\rho$ (consistent with the predictions of microscopic theories) which can hopefully be constrained by comparing theoretical cooling curves with the observations.

Superfluidity of neutrons and/or protons in neutron-star cores affects the heat capacity of nucleons and reduces neutrino reactions (Urca and nucleon-nucleon bremsstrahlung processes) involving superfluid nucleons (as reviewed, e.g., by Yakovlev et al. 1999). Moreover, superfluidity initiates an additional neutrino emission mechanism associated with Cooper pairing of nucleons (Flowers et al. 1976). All these effects of superfluidity are incorporated into our cooling code.

In our calculations we adopt one model of strong superfluidity of protons (with the maximum of $T_{\mathrm{cp}}(\rho)$ about $T_{\mathrm{cp}}^{\max } \approx$ $7 \times 10^{9} \mathrm{~K}$ ) and several models of moderate superfluidity of neutrons (with $T_{\mathrm{cn}}^{\max } \sim 6 \times 10^{8} \mathrm{~K}$ ) in a neutron-star core. These models are phenomenological but consistent with the results of microscopic theories. A pair of models: proton superfluidity 1 and neutron superfluidty nt 1 is plotted in the left panel of Fig. 1.

Strong proton superfluidity is required to slow down the cooling of low-mass stars, $M \lesssim 1.1 M_{\odot}$, whose central densities are $\rho_{\mathrm{c}} \lesssim 8 \times 10^{14} \mathrm{~g} \mathrm{~cm}^{-3}$. This scenario was suggested by Kaminker et al. (2001). In a low-mass star, one has $T_{\mathrm{c}}(\rho) \gtrsim$ $3 \times 10^{9} \mathrm{~K}$ everywhere in the core. Proton superfluidity occurs at the early cooling stage ( $t \lesssim 1 \mathrm{yr}$ ) and suppresses modified Urca processes of neutrino emission as well as neutrino generation in proton-proton and proton-neutron collisions. Neutrino emission due to Cooper pairing of protons is switched on too early and becomes inefficient in the middle-aged neutron stars we are interested in. In contrast, the adopted neutron superfluidity is too weak in low-mass stars (the left panel of Fig. 1) to appear at the neutrino cooling stage. This superfluidity does not suppress the neutrino emission in neutron-neutron collisions which becomes the leading mechanism of neutrino cooling. It is much weaker than the modified Urca process (which would be dominant in non-superfluid stars). As a consequence, the cooling curves of low-mass stars go noticeably higher than the universal cooling curve of non-superfluid stars. Actually, these cooling curves also merge into one almost universal curve, which is independent of the equation of state in a stellar core and of the exact behaviour of $T_{\mathrm{cp}}(\rho)$ (Kaminker et al. 2002). This upper curve 1 allows one to explain observations of the neutron stars hottest for their age (RX J0822-4300, 1E 1207.4-5209, PSR B1055-52, RX J0720.4-3125) as cooling low-mass neutron stars.

Now we come to observations of the neutron stars coldest for their age (first of all, PSR J0205+6449, the Vela pulsar, and Geminga). It has been widely proposed to interpret these objects as rather massive neutron stars with the neutrino emission enhanced by the direct Urca process in nucleon cores (or by similar processes in pion-condensed, kaon-condensed or quark cores). We will show that the coldest objects can be explained without invoking these mechanisms by tuning the model of moderate neutron superfluidity at $\rho \gtrsim 8 \times 10^{14} \mathrm{~g} \mathrm{~cm}^{-3}$. Let us consider the most massive neutron star $\left(1.994 M_{\odot}\right.$, curve 8$)$ in Fig. 1. Its central density is higher than the density at which neutron superfluidity nt 1 dies out. When the internal temperature of the star becomes lower than the maximum critical temperature of neutron superfluidity, the neutrino emission due to Cooper pairing of neutrons switches on and becomes a powerful neutrino emission mechanism, which can be about two orders of magnitude more efficient than the modified Urca process in a non-superfluid star (see Sect. 4). This emission produces enhanced cooling (attributed to direct Urca or similar processes in previous calculations). The enhancement is not very strong (e.g., the direct Urca process in a nucleon stellar core would further enhance the neutrino luminosity by about 4-5 orders of magnitude). However, even this not very strong enhancement is sufficient to explain the observations of the coldest neutron stars (particularly, PSR J0205+6449, the Vela and Geminga pulsars). Evidently, all neutron stars with $\rho_{\mathrm{c}} \gtrsim 2 \times 10^{15} \mathrm{~g} \mathrm{~cm}^{-3}$ (in our model) will cool nearly as fast as the $1.994 M_{\odot}$ star in Fig. 1.

Therefore, we come to three distinct classes of cooling neutron stars (similar to those described by Kaminker et al. 2002 for the case of enhanced cooling due to the direct Urca process). The first class contains low-mass, very slowly cooling stars (curve 1 in the right panel of Fig. 1). Another class contains high-mass stars with enhanced cooling (curve 8). Finally, there is a class of medium-mass neutron stars (curves 2-6) which show intermediate cooling. Their cooling curves fill in the space between the upper curve for low-mass stars and the lower curve for high-mass stars. These curves explain the observations of PSR B1706-44, PSR J0538+2817, and RX J1856.4-3754.

\section{Cooper-pairing neutrino emission as a fast-cooling agent}

Let us give a simple explanation of the computer results on enhanced neutrino emission due to Cooper pairing of neutrons. We start from the expression for the neutrino emissivity $Q_{\mathrm{CP}}$ due this process (e.g., Eq. (236) in Yakovlev et al. 2001). It can be written as

$Q_{\mathrm{CP}}(\rho, T)=q(\rho, T) F(\tau)$,

where

$$
\begin{aligned}
q(\rho, T) \approx & 1.17 \times 10^{21}\left(\frac{m_{N}^{*}}{m_{N}}\right)\left(\frac{p_{\mathrm{F}}}{m_{N} c}\right) \\
& \times T_{9}^{7} \mathcal{N}_{v} a_{N} \operatorname{erg~cm} \mathrm{cm}^{-3} \mathrm{~s}^{-1},
\end{aligned}
$$

$T \equiv T_{9} \times 10^{9} \mathrm{~K}$ is the internal stellar temperature, $m_{N}$ is the bare nucleon $\left(N=n\right.$ or $p$ ) mass, $m_{N}^{*}$ is the nucleon effective mass in dense matter, $p_{\mathrm{F}}$ is the nucleon Fermi momentum, $a_{N}$ is a dimensionless constant combined of squared weak-interaction constants of vector and axial-vector nucleon currents, $\mathcal{N}_{v}=3$ is the number of neutrino flavors, and $F(\tau)$ is a function of $\tau=T / T_{\mathrm{c}}$. The constant $a_{N}$ depends on nucleon species and pairing type, while $F(\tau)$ depends on pairing type. We have $a_{n}=4.17$ for the triplet-state neutron pairing under discussion. This value can be renormalized by many-body effects 
(for instance, the renormalization of the axial-vector constant was considered by Carter \& Prakash 2002). However, theoretical cooling curves are not very sensitive to the exact value of $a_{n}$, and we use the non-renormalized value. The analytic fit expression for $F(\tau)$ is presented, for instance, by Yakovlev et al. (2001). Let us recall that $F(\tau) \approx 4.71(1-\tau)$ just after superfluidity onset (immediately after $T$ falls below $T_{\mathrm{c}}$ ) and $F(\tau) \approx 1.27 \tau^{-6} \exp (-2.376 / \tau)$ at $\tau \ll 1$. Thus, the emissivity $Q_{\mathrm{CP}}(\rho, T)$ is exponentially suppressed at $T \ll T_{\mathrm{c}}$.

For our qualitative analysis in this section we employ the simplest dependence of the neutron critical temperature on distance $r$ from the stellar center:

$T_{\mathrm{cn}}(r)=T_{\mathrm{cm}}\left\{1-\frac{\left(r-r_{\mathrm{m}}\right)^{2}}{\left(\Delta r_{\mathrm{m}}\right)^{2}}\right\}$

at $\left|r-r_{\mathrm{m}}\right|<\Delta r_{\mathrm{m}}$ (with the maximum $T_{\mathrm{cm}}=T_{\mathrm{cn}}^{\max }$ at $r=r_{\mathrm{m}}$ ), and $T_{\mathrm{cn}}=0$ at $\left|r-r_{\mathrm{m}}\right| \geq \Delta r_{\mathrm{m}}$.

Neglecting, for simplicity, general relativistic effects and assuming an isothermal stellar core at a temperature $T<T_{\mathrm{cm}}$, the neutrino luminosity $L_{\mathrm{CP}}$ due to Cooper pairing of neutrons can be written as

$L_{\mathrm{CP}}=4 \pi \int_{r_{1}}^{r_{2}} r^{2} Q_{\mathrm{CP}} \mathrm{d} r$.

Here, $r_{1}$ and $r_{2}$ restrict the superfluid layer, where $T<T_{\mathrm{cn}}$ and the neutrino process in question is allowed. To be specific, let us assume that the widest superfluid layer (which is realized at $T=0$ and extends from $r_{\mathrm{m}}-\Delta r_{\mathrm{m}}$ to $r_{\mathrm{m}}+\Delta r_{\mathrm{m}}$ ) falls entirely in the neutron star core.

The factor $F(\tau)$ in the emissivity $Q_{\mathrm{CP}}$, Eq. (1), is a more rapidly varying function of $r$ than $q(\rho, T)$. Thus we can set $r=r_{\mathrm{m}}$ and $q(\rho, T)=q\left(\rho_{\mathrm{m}}, T\right)$ (with $\left.\rho_{\mathrm{m}}=\rho\left(r_{\mathrm{m}}\right)\right)$ in all functions under the integral but in $F(\tau)$. A simple replacement of the integration variable leads to

$$
\begin{aligned}
L_{\mathrm{CP}} & =8 \pi r_{\mathrm{m}}^{2} \Delta r_{\mathrm{m}} q\left(\rho_{\mathrm{m}}, T\right) \tau_{\mathrm{m}} \ell\left(\tau_{\mathrm{m}}\right), \\
\ell(\tau) & =\frac{1}{2} \int_{\tau}^{1} \frac{\mathrm{d} \tau^{\prime} F\left(\tau^{\prime}\right)}{\tau^{\prime 3 / 2} \sqrt{\tau^{\prime}-\tau}}
\end{aligned}
$$

where $\tau_{\mathrm{m}}=T / T_{\mathrm{cm}}$. The integration can be done numerically; the appropriate analytic fit (for triplet-state neutron pairing) is

$$
\begin{aligned}
\ell(\tau)= & (1-\tau)^{3 / 2}\left[3.844(1-\tau)+3.142 \tau^{2}\right. \\
& \left.+13.99 \tau(1-\tau)+\frac{25.4 \tau^{2.5}(1-\tau)^{2}}{\left((\tau-0.2493)^{2}+0.03694\right)^{0.7}}\right] .
\end{aligned}
$$

Evidently, the luminosity $L_{\mathrm{CP}}$ vanishes in a hot star where $T>T_{\mathrm{cm}}$ and neutron superfluidity is absent. It switches on as $T$ falls below $T_{\mathrm{cm}}$; it grows almost linearly while $T$ decreases to $\sim 0.8 T_{\mathrm{cm}}$; it reaches a maximum at $T=0.792 T_{\mathrm{cm}}$ (with $\tau \ell(\tau)=0.792 \ell(0.792)=0.481$ ) and then decreases. At the increasing and maximum-luminosity stage, $L_{\mathrm{CP}}$ is collected from a superfluid spherical stellar layer in the vicinity of the maximum critical temperature, $r \approx r_{\mathrm{m}}$. This creates a splash of neutrino emission associated with Cooper pairing of neutrons.

For typical values of the parameters, the maximum value of $L_{\mathrm{CP}}$ can be one to two orders of magnitude higher than the

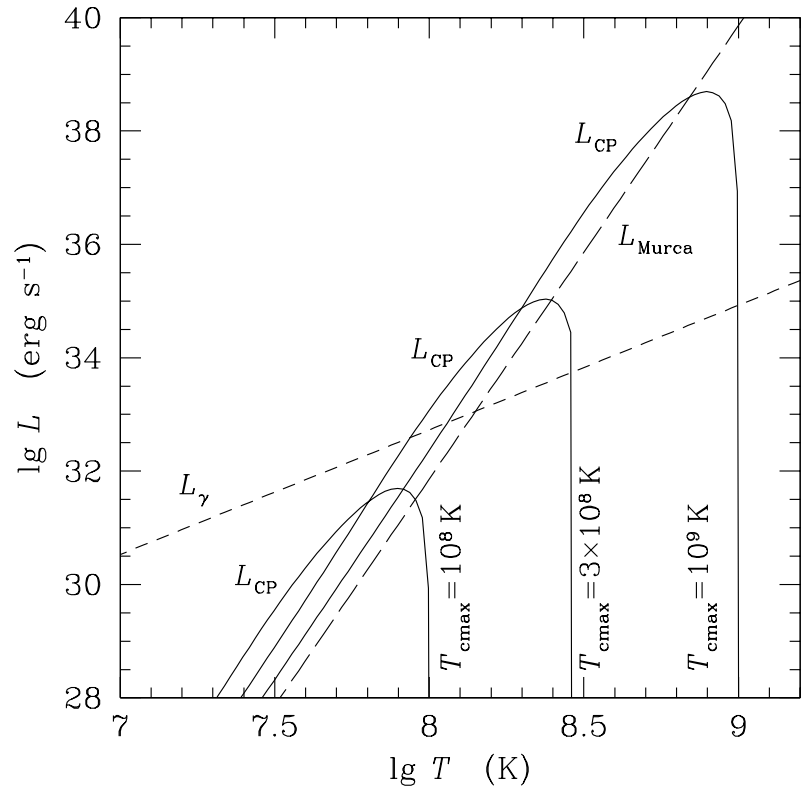

Fig. 2. A sketch of neutrino luminosities produced by the modified Urca process $\left(L_{\text {Murca }}\right)$ and the Cooper pairing process $\left(L_{\mathrm{CP}}\right)$ as well as of the photon luminosity $L_{\gamma}$ of a neutron star versus internal temperature $T$ for three models of neutron superfluidity in the stellar core with $T_{\mathrm{cn}}^{\max }=10^{8}, 3 \times 10^{8}$ and $10^{9} \mathrm{~K}$.

neutrino luminosity $L_{\text {Murca }}$ of a non-superfluid star (with forbidden direct Urca process). This is demonstrated in Fig. 2 using a toy model of cooling neutron stars described by Yakovlev \& Haensel (2003) - there is no need to employ accurate models in this section. The parameters of the neutron-star model presented in the figure are: $M=1.16 M_{\odot}, R=12 \mathrm{~km}$, $\rho_{\mathrm{c}}=8 \times 10^{14} \mathrm{~g} \mathrm{~cm}^{-3}, r_{\mathrm{m}}=\Delta r_{\mathrm{m}}=5 \mathrm{~km}$. The three superfluidity models $\left(T_{\mathrm{cn}}(r)\right)$ are self-similar and differ by the values of $T_{\mathrm{cm}}=10^{8}, 3 \times 10^{8}$, and $10^{9} \mathrm{~K}$. Three solid lines show the Cooper-pairing neutrino luminosity $L_{\mathrm{CP}}$ calculated from Eqs. (4)-(7) for the three models of neutron superfluidity. Since $L_{\text {Murca }} \propto T^{8}$ and $L_{\mathrm{CP}}^{\max } \propto T^{7}$, the Cooper-pairing luminosity is more competitive at weaker superfluidity (lower $T_{\mathrm{cm}}$ ). However, at $T_{\mathrm{cm}} \lesssim 2 \times 10^{8} \mathrm{~K}$ this luminosity becomes lower than the photon thermal luminosity of the star (Fig. 2) which makes it insignificant for stellar cooling. Note that, for realistic parameters, $L_{\mathrm{CP}}$ is much smaller than the neutrino luminosity due to the direct Urca process in a non-superfluid star (if the direct Urca process is open).

The decreasing part of $L_{\mathrm{CP}}(T)$ is even more fascinating. We have $\ell(\tau) \approx 3.84$ as $\tau \rightarrow 0$, resulting in the scaling relation

$L_{\mathrm{CP}} \propto \Delta r_{\mathrm{m}} T^{8} / T_{\mathrm{cm}}$,

which becomes sufficiently accurate at $T \lesssim 0.6 T_{\mathrm{cm}}$. This neutrino emission is actually produced from two thin spherical shells (near $r=r_{1}$ and $r=r_{2}$ ), where $T$ is just below $T_{\mathrm{c}}(r)$. The widths of these shells are proportional to $T$, which explains the power-law $T^{8}$ (instead of the exponential decrease of the emissivity $Q_{\mathrm{CP}}(\rho, T)$ in a local element of superfluid matter). Therefore, the decreasing part of the Cooper-pairing neutrino luminosity has the same temperature dependence as all slow neutrino emission mechanisms (modified Urca, 

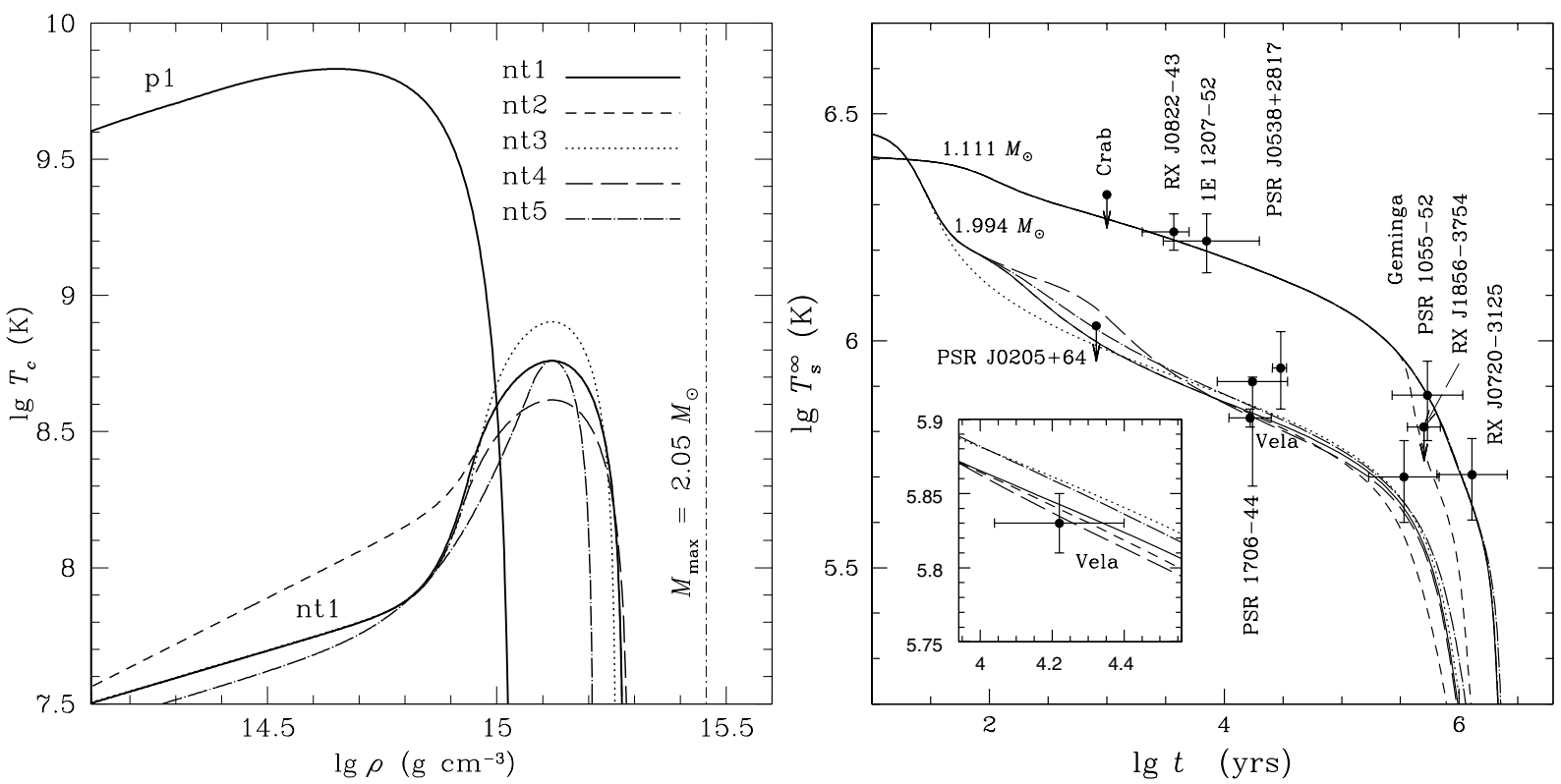

Fig. 3. Left: One model p1 for proton superfluidity and five models nt $1-n t 5$ for neutron superfluidity in a neutron-star core. Right: Cooling curves of low-mass $\left(1.111 M_{\odot}\right)$ and high-mass $\left(1.994 M_{\odot}\right)$ stars with model p1 proton superfluidity and one of the models of neutron superfluidity from the left panel, compared with the observations. Cooling of the low-mass star is insensitive to selected models of neutron superfluidity (except for model nt2 at $t>300 \mathrm{kyr}$ ). The insert shows the comparison of cooling curves of the high-mass star with observations of the Vela pulsar in more detail.

nucleon-nucleon bremsstrahlung) in non-superfluid cores of stars with forbidden direct Urca process. In other words, the superfluidity suppresses the neutrino emission available in nonsuperfluid stars but initiates the Cooper-pairing neutrino emission in such a way that it acts as a new nonsuppressed neutrino cooling mechanism. Moreover, the new emission can be more intense than that in a non-superfluid star and provide enhanced cooling. This important feature appears in realistic models of cooling neutron stars with density-dependent critical temperatures $T_{\mathrm{c}}(\rho)$ (and does not appear in the models with densityindependent $T_{\mathrm{c}}$ ). In particular, it implies that, once $L_{\mathrm{CP}}$ takes on leadership in competition with $L_{\text {Murca }}$ just after the superfluidity onset, it will not lose it during the subsequent evolution (especially because $L_{\text {Murca }}$ is actually suppressed by superfluidity, which is not taken into account in Fig. 2). This is clearly seen from Fig. 2.

Let us add that at $T \ll T_{\mathrm{cm}}$ we can obtain a better formula for $L_{\mathrm{CP}}$ than Eq. (4), without employing the specific $T_{\mathrm{c}}(r)$ profile, Eq. (3). It is sufficient to start from Eq. (4) and notice that the main contribution into $L_{\mathrm{CP}}$ comes from two thin shells, at $r \approx r_{1}$ and $r \approx r_{2}$, where $T_{\mathrm{c}}(r) \approx T$. In each shell, the gradient $D=\mathrm{d} T_{\mathrm{c}}(r) / \mathrm{d} r$ can be taken constant. Then we get

$L_{\mathrm{CP}}=8 \pi\left[r_{1}^{2} H_{1} q\left(\rho_{1}, T\right)+r_{2}^{2} H_{2} q\left(\rho_{2}, T\right)\right] \ell(0)$,

where $H_{1}=T /\left|D_{1}\right|$ and $H_{2}=T /\left|D_{2}\right|$ are characteristic widths of our shells, $\rho_{1}=\rho\left(r_{1}\right), \rho_{2}=\rho\left(r_{2}\right)$, and $\ell(0)=3.84$. Strictly speaking, $r_{1}, r_{2}, \rho_{1}, \rho_{2}, D_{1}$, and $D_{2}$ depend slightly on $T$, but this dependence can be regarded as parametric. It is easy to verify that if $T_{\mathrm{c}}(r)$ is given by Eq. (3) at $T \ll T_{\mathrm{cm}}$ and $\Delta r_{\mathrm{m}} \ll r_{\mathrm{m}}$, our new expression for $L_{\mathrm{CP}}$ coincides with Eq. (5). Equation (5) is expected to be useful just after the superfluidity onset, at $0.6 T_{\mathrm{cm}} \lesssim T<T_{\mathrm{cm}}$ (where the parabolic $T_{\mathrm{c}}(r)$ dependence may be a good approximation), while Eq. (9) is more exact at lower $T$. Both equations enable one to incorporate the Cooperpairing neutrino emission in simplified cooling models (like a toy model of Yakovlev \& Haensel 2003), useful for understanding the main features of neutron star cooling without complicated cooling codes.

The above analysis is valid as long as $T_{\mathrm{c}}(\rho)$ vanishes in the stellar interior. If it does not, there is a minimum value $T_{\mathrm{c}}^{\mathrm{min}}$ of $T_{\mathrm{c}}(\rho)$, and $L_{\mathrm{CP}}$ will become exponentially suppressed at $T \ll T_{\mathrm{c}}^{\mathrm{min}}$.

\section{Testing the cooling scenario and discussion}

After clarifying the efficiency of the Cooper-pairing neutrino emission let us return to the cooling scenario described in Sect. 3. As we have already mentioned, the scenario is rather insensitive to a specific model of proton superfluidity (required to raise the surface temperature of low-mass stars in order to explain the observations of the sources hottest for their ages). The only serious constraint on the proton pairing is that $T_{\mathrm{cp}}(\rho)$ should be high $\left(\nexists 3 \times 10^{9} \mathrm{~K}\right)$ in the cores of low-mass stars.

However, the constraints on the neutron critical temperature $T_{\mathrm{cn}}(\rho)$ in a stellar core should be really strong. This is illustrated in Fig. 3. The left panel displays the critical temperatures of our proton superfluidity model (p1) and five neutron superfluidity models (nt1-nt5), including our basic model nt1 used in Sect. 3. The right panel shows cooling curves of a low-mass $\left(1.111 M_{\odot}\right)$ star and a high-mass $\left(1.994 M_{\odot}\right)$ star. Any curve is calculated for model p1 of the proton superfluidity and one model of the neutron superfluidity from the left panel of Fig. 3. Any observational point between an upper curve and a lower 
curve can be explained by a given superfluid model. The constraints on neutron superfluidity are as follows.

(1) Neutron superfluidity should be weak in low-mass stars. In our case (for the equation of state of Douchin \& Haensel 2001) this means that $T_{\text {cn }}(\rho) \lesssim 2 \times 10^{8} \mathrm{~K}$ at $\rho \lesssim 8 \times 10^{14} \mathrm{~g} \mathrm{~cm}^{-3}$. Under this condition neutron superfluidity does not affect the cooling (at least at the neutrino cooling stage) of low-mass stars $\left(M \lesssim 1.1 M_{\odot}\right)$ and does not violate our interpretation of the sources hottest for their age (first of all, RX J0822-4300 and PSR B1055-52). Accordingly, all five cooling curves (for superfluids nt1-nt5) of low-mass stars merge in one upper (solid) cooling curve in Fig. 3. The only exception is provided by model nt 2 with highest pre-peak $T_{\mathrm{cn}}(\rho)$ among models nt $1-\mathrm{nt} 5$. In a low-mass star this superfluidity occurs at $t \gtrsim 300 \mathrm{kyr}$. The Cooper-pairing neutrino emission and reduced heat capacity of neutrons noticeably accelerate the cooling at this late stage (the upper short-dashed curve).

(2) The neutron superfluidity should be moderate at $\rho \gtrsim$ $10^{15} \mathrm{~g} \mathrm{~cm}^{-3}$, with the peak maximum $T_{\mathrm{cn}}^{\max } \sim 6 \times 10^{8} \mathrm{~K}$ (model nt1 in Fig. 3, the solid curve). In this case it switches on just in time to initiate the enhanced cooling in a high-mass star. Its level is sufficient to explain the observations of the neutron stars coldest for their ages (first of all, PSR J0205+6449 and the Vela pulsar). The asymptotic neutrino-cooling regime given by the scaling expression (8) is realized at $t \gtrsim(1-10) \mathrm{kyr}$. If $T_{\mathrm{cn}}^{\max }$ were slightly higher than $6 \times 10^{8} \mathrm{~K}$ (model nt $3, T_{\mathrm{cn}}^{\max }=8 \times$ $10^{8} \mathrm{~K}$, the dotted curve), the Cooper-pairing neutrino emission will start operating in a younger massive star but becomes less efficient at $t \sim 10 \mathrm{kyr}$, which is less favorable for explaining the observations of the Vela pulsar. This cooling behaviour is naturally explained by the scaling (8). If $T_{\mathrm{cn}}^{\max }$ were slightly lower than $6 \times 10^{8} \mathrm{~K}$ (model nt $4, T_{\mathrm{cn}}^{\max }=4 \times 10^{8} \mathrm{~K}$, the longdashed curve), the Cooper-pairing neutrino emission will start operating too late which would violate the interpretation of the observations of PSR J0205+6449.

(3) The results are also sensitive to the width of the peak of the $T_{\mathrm{cn}}(\rho)$ curve. For instance, retaining the peak maximum of $6 \times 10^{8} \mathrm{~K}$ but making the peak narrower (model nt5, the dotdashed curve) will reduce the neutrino emissivity due to neutron pairing, raise the temperature of the massive star and complicate the interpretation of the Vela pulsar (again, in agreement with the scaling (8)). However, the cooling curves are rather insensitive to the exact position of the $T_{\mathrm{cn}}(\rho)$ maximum. We can slightly shift the maximum to higher or lower $\rho$ (confining the peak within the kernel of a massive star) but these shifts will not change the cooling curves of massive stars (such tests are not shown in Fig. 3). However, the shift of the maximum to $\rho \lesssim 8 \times 10^{14} \mathrm{~g} \mathrm{~cm}^{-3}$ would cause the enhanced cooling of lowmass stars. The cooling curves of low-mass stars would become close to those of high-mass stars which would violate the interpretation of the observations of neutron stars hottest for their ages (see item (1)).

This discussion shows that the cooling curve of a massive neutron star implying model nt 1 of neutron superfluidity is close to the lowest cooling curve (in the scenario, where the cooling is enhanced by Cooper-pairing neutrino emission). Observations of cold neutron stars, PSR J0205+6449 and the Vela pulsar, provide excellent tests for this scenario. If these pulsars were noticeably colder we would be unable to explain them within our scheme. Notice that the upper limit of the surface temperature of PSR J0205+6449 was inferred from observations (Slane et al. 2002) using the blackbody spectrum of surface emission. If this pulsar has a hydrogen atmosphere, the upper limit on $T_{\mathrm{s}}^{\infty}$ could be expected to be about twice lower than for the blackbody case. In that case we would be unable to explain this source within the proposed scenario.

Although we have used one equation of state of dense matter (Douchin \& Haensel 2001) we would obtain similar results for other equations of state which forbid direct Urca processes (and other similar processes of fast neutrino cooling) in neutron star cores. Taking different equations of state would lead to attributing different masses to the same sources (Fig. 1); a similar problem has been discussed by Kaminker et al. (2002).

In addition, we could take an equation of state in the stellar core which opens the direct Urca process at the highest densities (in the central kernels of the most massive stable neutron stars; similar to the equation of state of Akmal \& Pandharipande 1997). Applying the same model of nucleon superfluidity as in Fig. 1, we would get five types of cooling neutron stars (instead of three). Three types would be the same as those mentioned in Sect. 3: low-mass, very slowly cooling stars; massive stars whose cooling is enhanced by Cooperpairing neutrino emission; and medium-mass stars whose cooling is intermediate. In addition, we would have: the most massive neutron stars showing very fast cooling via the direct Urca process; and stars whose cooling is intermediate between that enhanced by the Cooper-pairing neutrino emission and that enhanced by the direct Urca process. The transition from the Cooper-pairing neutrino cooling to the direct-Urca cooling with increasing mass $M$ will be very sharp and the number of intermediate-cooling sources will be small. The maximummass neutron stars would be extremely cold $\left(T_{\mathrm{s}}^{\infty} \sim 2 \times 10^{5} \mathrm{~K}\right.$ at $t \sim 10 \mathrm{kyr}$ ), about the same as discussed, e.g., by Kaminker et al. (2002). A discovery of such stars would definitely indicate the operation of the direct Urca process in their cores. Indirect evidence of their existence is provided by the non-detection of neutron stars in some supernova remnants (Kaplan et al. 2004).

Note that the cooling of neutron stars can also be affected by singlet-state superfluidity of neutrons in inner stellar crusts, by the presence of surface layers of light (accreted) elements, and by stellar magnetic fields (e.g., Potekhin et al. 2003; Geppert et al. 2004). These effects can be especially important in low-mass stars. We have neglected them in the present paper since we have mainly focused on enhanced cooling of massive stars but we will consider them in a future publication.

\section{Conclusions}

We have proposed a new scenario of cooling of isolated neutron stars. We have shown that the present observational data on thermal emission from isolated middle-aged neutron stars can be explained assuming that neutron star cores are composed of neutrons, protons and electrons (and possibly muons) with forbidden direct Urca process of neutrino emission. In our scenario, enhanced neutrino emission, which is required for the interpretation of the neutron stars coldest for their age, is 
provided by the neutrino process associated with Cooper pairing of neutrons. We have shown that the neutrino luminosity due to this process (at internal temperatures $T \lesssim 0.6 T_{\mathrm{cn}}^{\max }$ ) behaves as $T^{8}$. In this way it "mimics" the neutrino luminosity produced either by modified Urca processes or by nucleonnucleon bremsstrahlung processes in non-superfluid stars, but it can be one to two orders of magnitude higher. The proposed cooling scenario imposes very stringent constraints on the density dependence of neutron-pairing temperature $T_{\mathrm{cn}}(\rho)$. The constraints are the result of the comparison of the cooling theory with the two most important "testing sources", PSR J0205+6449 and the Vela pulsar (Sect. 5). This scenario is the first one in which moderate superfluidity and associated neutrino emission are helpful for explaining the data (cf. with previous cooling scenarios, where moderate superfluidity violated the interpretation of the observations, e.g., Kaminker et al. 2002).

Our interpretation implies the presence of strong proton superfluidity and moderate neutron superfluidity in neutron star cores (Sect. 3). We need the proton superfluidity to explain the observations of the neutron stars hottest for their age, and the neutron superfluidity to explain the observations of the stars coldest for their age. However, as has been demonstrated by Gusakov et al. (2004), cooling curves are not too sensitive to exchanging neutron and proton superfluidities $\left(T_{\mathrm{cp}}(\rho) \rightleftharpoons\right.$ $\left.T_{\text {cn }}(\rho)\right)$ in neutron-star cores. Therefore, we would also be able to explain the observational data in the scenario with strong neutron superfluidity and moderate proton superfluidity in stellar cores.

We need strong superfluidity to suppress the modified Urca process in low-mass stars, raise the surface temperature of these stars and explain the observations of the neutron stars hotter for their age. In fact, we can raise the temperature of low-mass middle-aged neutron stars by assuming the presence of surface layers of light (accreted) elements. The mass of light elements may decrease with time, e.g., due to diffusive nuclear burning (Chang \& Bildsten 2003), which gives additional freedom for regulating the cooling. In this way, the presence of strong (proton or neutron) superfluidity in a neutron star core is not vitally important for our interpretation. We will show this in a future publication. However, the presence of moderate superfluidity (of neutrons or protons) with a tuned density dependence of critical temperature (Sect. 5) is crucial for this scenario, where this tuned dependence is combined with the remarkable simplicity of the equation of state of neutron-star cores (nucleon composition with forbidden direct Urca process). We hope that this scenario can be taken into consideration along with many other scenarios (reviewed or proposed, e.g., by Page 1998a,b; Tsuruta et al. 2002; Khodel et al. 2004; Blaschke et al. 2004; Yakovlev \& Pethick 2004). The correct scenario should be selected in future observations of neutron stars combined with new advanced theoretical results.

After this paper was prepared for submission we became aware of the paper of Page et al. (2004). These authors give a detailed consideration of enhanced cooling via neutrino emission due to Cooper pairing of neutrons in neutron-star cores composed of nucleons with forbidden direct Urca process. The idea to enhance the cooling by Cooper-pairing neutrino emission is the same as in our paper, but its realization is different. Particularly, Page et al. (2004) use a set of superfluidity models obtained from microscopic theories. Their main models for neutron superfluidity in a stellar core (for, instance, model (a) in their Fig. 9) have too high peak temperatures $T_{\mathrm{cn}}^{\max } \gtrsim 10^{9} \mathrm{~K}$ and too high $T_{\mathrm{cn}}(\rho)$ at the pre-peak densities to explain the observations of PSR J0205+6449 and the Vela pulsar and to obtain a pronounced dependence of cooling curves on neutron star mass. In contrast, our $T_{\mathrm{cn}}(\rho)$ models are phenomenological but by tuning them we obtain a noticeable dependence of the cooling on $M$. It enables us to explain all the data by one model of nucleon superfluidity (even neglecting the effect of accreted envelopes).

Note added in proof. After this paper had been accepted for publication we became aware of the paper by Slane et al. (Slane, P., Helfand, D. J., van der Swaluw, E., \& Murray, S. S. 2004, ApJ, submitted [arXiv: astro-ph/0405380]). The authors estimate $T_{\mathrm{s}}^{\infty}=(9.3 \pm 0.3) \times 10^{5} \mathrm{~K}$ for PSR J0205+6449 using the hydrogen atmosphere model. This estimate turns out to be higher than we have expected in Sect. 5 and is marginally consistent with the cooling of the massive neutron star discussed in Sect. 3 (Fig. 1).

Acknowledgements. We are grateful to A. Y. Potekhin for helpful discussions. This work has been supported partly by the RFBR, grants 02-02-17668 and 03-07-90200, the RLSS, grant 1115.2003.2, and by the INTAS, grant YSF 03-55-2397.

\section{References}

Akmal, A., \& Pandharipande, V. R. 1997, Phys. Rev. C, 56, 2261

Arendt, R. G., Dwek, E., \& Petre, R. 1991, ApJ, 368, 474

Bejger, M., Yakovlev, D. G., \& Gnedin, O. Y. 2003, Acta Phys. Polonica B34, 221

Blaschke, D., Grigorian, H., \& Voskresensky, D. N. 2004, A\&A, submitted [arXiv:astro-ph/0403170]

Brisken, W. F., Thorsett, S. E., Golden, A., \& Goss, W. M. 2003, ApJ, 593, L89

Burwitz, V., Haberl, F., Neuhäuser, R., et al. 2003, A\&A, 399, 1109

Carter, G. W., \& Prakash, M. 2002, Phys. Lett. 525B, 249

Chang, P., \& Bildsten, L. 2003, ApJ, 585, 464

Cropper, M., Haberl, M., Zane, S., \& Zavlin, V. E. 2004, MNRAS, submitted [arXiv:astro-ph/0403380]

Douchin, F., \& Haensel, P. 2001, A\&A, 380, 151

Flowers, E. G., Ruderman, M., \& Sutherland, P. G. 1976, ApJ, 205, 541

Geppert, U., Küker, M., \& Page, D. 2004, A\&A, submitted [arXiv: astro-ph/0403441]

Gnedin, O. Y., Yakovlev, D. G., \& Potekhin, A. Y. 2001, MNRAS, 324,725

Gusakov, M. E., Kaminker, A. D., Yakovlev, D. G., \& Gnedin, O. Y. 2004, Astron. Lett., 30(10), to appear

Kaminker, A. D., Haensel, P., \& Yakovlev, D. G. 2001, A\&A, 373, L17

Kaminker, A. D., Yakovlev, D. G., \& Gnedin, O. Y. 2002, A\&A, 383, 1076 
Kaplan, D. L., Kulkarni, S. R., van Kerkwijk, M. H., \& Marshall, H. L. 2002, ApJ, 570, L79

Kaplan, D. L., Frail, D. A., Gaensler, B. M., et al. 2004, ApJ, submitted [arXiv: astro-ph/0403313]

Khodel, V. A., Clark, J. W., Takano, M., \& Zverev, M. V. 2004, Phys. Rev. Lett., submitted [arXiv: astro-ph/0402514]

Kramer, M., Lyne, A. G., Hobbs, G., et al. 2003, ApJ, 593, L31

Lattimer, J. M., Pethick, C. J., Prakash, M., \& Haensel, P. 1991, Phys. Rev. Lett., 66, 2701

Lombardo, U., \& Schulze, H.-J. 2001, in Physics of Neutron Star Interiors, ed. D. Blaschke, N. K. Glendenning, \& A. Sedrakian (Berlin: Springer), 30

Lyne, A. G., Pritchard, R. S., Graham-Smith, F., \& Camilo, F. 1996, Nature, 381, 497

McGowan, K. E., Zane, S., Cropper, M., et al. 2004, ApJ, 600, 343

Motch, C., Zavlin, V. E., \& Haberl, F. 2003, A\&A, 408, 323

Page, D. 1998a, in The Many Faces of Neutron Stars, ed. R. Buccheri, J. van Paradijs, \& M. A. Alpar (Dordrecht: Kluwer), 539

Page, D. 1998b, in Neutron Stars and Pulsars, ed. N. Shibazaki, N. Kawai, S. Shibata, \& T. Kifune (Tokyo: Universal Academy Press), 183

Page, D., \& Applegate, J. H. 1992, ApJ, 394, L17

Page, D., Lattimer, J. M., Prakash, M., \& Steiner, A. W. 2004, ApJ, submitted [arXiv: astro-ph/0403657]

Pavlov, G. G., Zavlin, V. E., \& Sanwal, D. 2002, in 270 WE-Heraeus Seminar on Neutron Stars, Pulsars and Supernova Remnants, ed. W. Becker, H. Lesh, \& J. Trümper (MPE: Garching), 273

Pavlov, G. G., \& Zavlin, V. E. 2003, in Texas in Tuscany, XXI Texas Symposium on Relativistic Astrophysics, ed. R. Bandiera, R. Maiolino, \& F. Mannucci (Singapore: World Scientific Publishing), 319

Pavlov, G. G., Zavlin, V. E., Sanwal, D., Burwitz, V., \& Garmire, G. P. 2001, ApJ, 552, L129

Pons, J. A., Walter, F., Lattimer, J., et al. 2002, ApJ, 564, 981

Potekhin, A. Y., Yakovlev, D. G., Chabrier, G., \& Gnedin, O. Y. 2003, ApJ, 594, 404
Roger, R. S., Milne, D. K., Kesteven, M. J., Wellington, K. J., \& Haynes, R. F. 1988, ApJ, 332, 940

Schwenk, A., \& Friman, B. 2004, Phys. Rev. Lett., 92, 082501

Slane, P. O., Helfand, D. J., \& Murray, S. S. 2002, ApJ, 571, L45

Tsuruta, S., Teter, M. A., Takatsuka, T., Tatsumi, T., \& Tamagaki, R. 2002, ApJ, 571, L143

Walter, F. M. 2001, ApJ, 549, 433

Walter, F. M., \& Lattimer, J. M. 2002, ApJ, 576, L145

Weisskopf, M. C., O’Dell, S. L., Paerels, F., et al. 2004, ApJ, 601, 1050

Winkler, P. F., Tuttle, J. H., Kirshner, R. P., \& Irwin, M. J. 1988, in Supernova Remnants and the Interstellar Medium, ed. R. S. Roger, \& T. L. Landecker (Cambridge: Cambridge Univ. Press), 65

Yakovlev, D. G., \& Haensel, P. 2003, A\&A, 407, 259

Yakovlev, D. G., \& Pethick, C. J. 2004, ARA\&A, in press [arXiv:astro-ph/0402143]

Yakovlev, D. G., Levenfish, K. P., \& Shibanov, Yu. A. 1999, Physics Uspekhi, 42, 737

Yakovlev, D. G., Kaminker, A. D., Gnedin, O. Y., \& Haensel, P. 2001, Phys. Rept., 354, 1

Yakovlev, D. G., Gnedin, O. Y., Kaminker, A. D., \& Potekhin, A. Y. 2002, in 270 WE-Heraeus Seminar on Neutron Stars, Pulsars and Supernova Remnants, ed. W. Becker, H. Lesh, \& J. Trümper (MPE: Garching), 287

Zane, S., Haberl, F., Cropper, M., et al. 2002, MNRAS, 334, 345

Zavlin, V. E., \& Pavlov, G. G. 2002, in 270 WE-Heraeus Seminar on Neutron Stars, Pulsars and Supernova Remnants, ed. W. Becker, H. Lesh, \& J. Trümper (MPE: Garching), 263

Zavlin, V. E., \& Pavlov, G. G. 2003, Memorie della Societa' Astronomica Italiana, in press [arXiv: astro-ph/0312326]

Zavlin, V. E., Trümper, J., \& Pavlov, G. G. 1999, ApJ, 525, 959

Zavlin, V. E., Pavlov, G. G., \& Sanwal, D. 2004, ApJ, 606, 444

Zuo, W., Li, Z. H., Lu, G. C., et al. 2004 [nucl-th/0403026] 